\title{
A Joint-Diffused Inpainting Model for Underexposure Image Preserving the Linear Geometric Structure
}

\author{
Jiying WU, Qiuqi RUAN, Gaoyun AN \\ Institute of Information Science, Beijing Jiaotong University \\ e-mail: happywjy82@126.com,qqruan@center.njtu.edu.cn,gaoyun_an@126.com
}

Received: December 2007; accepted: April 2008

\begin{abstract}
To restore the underexposure image, an illumination compensation inpainting model which employs the joint-diffused partial differential equations (PDEs) is proposed. Firstly, the novel model compensates the illumination effect in multi-scaled underexposure images respectively. Secondly, the information in the fused compensated image is restored by PDEs which diffuse the geometric property and gray information into the target region simultaneously. Experimental results demonstrate that the novel model can properly restore scratches while compensating the illumination effect in underexposure image, and the joint-diffused PDEs which are employed in it lead to a better performance than the conventional PDE inpainting models.
\end{abstract}

Keywords: image inpainting, illumination compensation, partial differential equation (PDE), quotient image (QI).

\section{Introduction}

Image inpainting is used to restore scratches in damaged image. The scratch is chosen manually, and then the model automatically restores the geometric property and gray information in scratch based on the known image information.

Recently, many methods are proposed to inpaint images with different geometric structures. Processing the geometric structure directly could preserve the composite features in image (Malickas and Vitkus, 2001). Partial differential equation (PDE) which restores image information by anisotropic diffusion was widely used in natural image inpainting. Total variation (TV) and pure transportation model were firstly used in inpainting which diffuse image information along the gradient direction and isophotes direction respectively (Chan and Shen, 2001a; Bertalmio et al., 2001). The Euler's elastic function is adopted which restores image as curves (Chan et al., 2002). It diffuses along both the gradient direction and the isophotes direction. Experimental results proved the bi-directional diffused Euler equation inpainted image better. While Euler equation is a 4th order PDE, the numerical scheme of it is very complex and the processing time is long. Chan proposed a 3rd order PDE which also diffuses along two orthogonal directions (Chan and Shen, 2001b). The numerical scheme of it is simple, but there are 
undetermined coefficients. A TV-Stokes equation is proposed by Tai for inpainting (Tai et al., 2006). It diffuses the geometric directions firstly and then reconstructs image to fit the restored directions. Chan etc proposed a joint-diffused PDEs inpainting model which is also comprised by high-order PDEs (Ballester et al., 2001).

All above mentioned methods were focused on restoring the geometric structures in natural image. Different PDEs were used to properly reconnect the geometric features. While there is another kind of defect that needs to be restored in natural image. The natural image is often collected under low-level lighting condition or varied viewing points. Therefore some image information is defected and unperceived in the overexposure image or in the underexposure image ( $\mathrm{Li}$ and Jain, 2005). A highlight removal inpainting method is proposed in (Park and Lee, 2007), which restores the highlights in image under the constraint of illumination effect. Some transforming methods, such as the Log operator, are proposed to directly correct the illumination effect in image (Adini et al., 1997). But these methods change the gray contrast and even induce noises into the image. They could not restore the corrupted information caused by lighting effect.

In this paper, our research focuses on deriving a joint diffused inpainting model which is used for underexposure image. There are shadows in the underexposure images and the information in the shadows is too dark to perceive. To generate an image with a good visual perception, the model compensates the illumination effect and restores the target region simultaneously. The Quotient Image (QI) method is used to remove the lighting effects in image (Chen et al., 2006) and the joint-diffused PDEs are used to inpaint image. QI method enhances the image contrast and it does not induce any noisy information. The PDEs diffuse both the geometric property and gray information into the target region. Also the gray information is diffused along two orthogonal geometric directions to smoothly restore the image. So the visual perception of the processed result is good. The lighting effects, geometric structures and gray information are all well restored.

\section{The Proposed Model}

The whole processing procedure of the model proposed in this paper is shown in Fig. 1. The novel model is used to inpaint the underexposure image which has low-level illumination effect. So the main task of it is illumination normalization and joint-diffused inpaiting.

Firstly, it normalizes the illumination effect by using QI model. The Total Variation model with $\mathrm{L}^{1}$ norm is used to decompose the image into two parts: a large-scale part which has the diffuse reflections and a small-scale part which contains the intrinsic textured information. The large-scale part is normalized by the Histogram Equalization to adjust the contrast of image and enhance the meaningful information. The small-scale part is normalized by using QI to enhance the signals of intrinsic structures in the shadows. Lastly, a feature fusion method is adopted to fuse the multi-scaled properties.

After compensating the illumination effect, the model diffuses the geometric property and gray information into the target region to inpaint image. Along the gradient direction, pixel's gray level changes greatly. So the dynamic changing geometric property is 


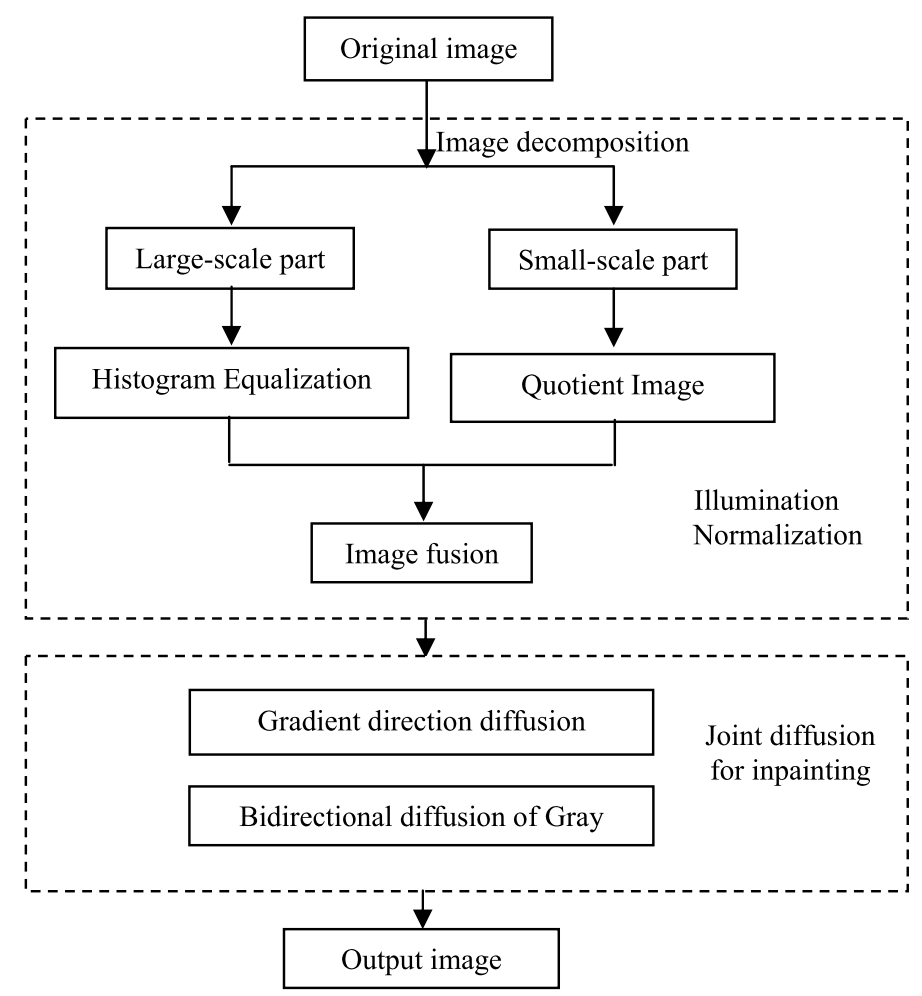

Fig. 1. The whole processing procedure.

preserved by diffusing the information about gradient directions. The gray information is diffused along two orthogonal geometric directions to smoothly restore the image. The diffusion along gradient direction uses the TV model which is morphological invariant, and the diffusion along isophotes direction uses the inviscid Helmholtz vorticity equation in fluid dynamics.

\subsection{Illumination Compensation}

The diffuse reflection in image could be defined by the Lambertian model as

$$
I=\rho S
$$

where $\rho$ is the albedo which is determined by the material and the geometric surface of the object, $S$ represents the lighting condition for image. Since the lighting effect in image changes slowly, $S$ represents the low-frequency part of image. In this paper, illumination effect is compensated in both the high-frequency and the low-frequency part. 
TV-L ${ }^{1}$ is an image decomposition model which decomposes the image into a largescale part $u$ and a small-scale part $v$ (Yin et al., 2005):

$$
I=u+v
$$

The large-scale part contains the image information about the lighting effect, region contour, and large geometric structure. So it represents the low-frequency part of image. The small-scale part contains the intrinsic geometric and textured information of image, so it often represents the high-frequency part. TV-L $\mathrm{L}^{1}$ processes image under the constraint of $\|\bullet\|_{1}-$ norm, and it has a scale-selecting property:

$$
J_{\lambda}[u]=\int|\nabla u| \mathrm{d} x+\lambda \int|I-u| \mathrm{d} x .
$$

To maintain the numerical stability of $\mathrm{TV}^{-} \mathrm{L}^{1}$ model, a disturbance $\varepsilon$ is added into the fidelity term of Eq. (3):

$$
J_{\lambda}[u]=\int|\nabla u| \mathrm{d} x+\lambda \int \sqrt{(I-u)^{2}+\varepsilon} \mathrm{d} x .
$$

By minimizing Eq. (4), the large-scale part $u$ which contains the lighting information is generated. The difference between $u$ and the original image $I$ is the small-scale part.

$$
v=I-u \text {. }
$$

According to the Lambertian model, the ratio of albedo between original image and the low-frequency part which is the QI has illumination invariant property. So QI is used to normalize the illumination effect in $v$ :

$$
\widehat{v}=I / u \text {. }
$$

After the quotient operation all the textured information in shadows is enhanced.

To adjust the contrast of image and enhance the meaningful information, histogram equalization is used in $u$. A flat histogram $H$ with gray level $K$ is created as

$$
H=\left[\begin{array}{llll}
1 & 1 & \ldots & 1
\end{array}\right]_{1 \times K} *\left(n^{2} / K\right)
$$

With the flat histogram $H$, a gray scale transformation $T(\bullet)$ is chosen to minimize:

$$
\left|h_{1}(T(k))-h_{0}(k)\right|
$$

where $h_{0}(\bullet)$ is the cumulative histogram of $u, h_{1}(\bullet)$ is the cumulative sum of $H$ for all intensities $k$. Minimization of Eq. (8) is subject to the constraints that the gray scale transformation must be monotonic and $h_{1}(T(\mu))$ cannot overshoot $h_{0}(\mu)$ by more than half the 
distance between the histogram counts at $\mu$. After getting the gray scale transformation, $u$ is processed to enhance its contrast as

$$
\overparen{u}=T(u) .
$$

The meaningful image information is enhanced in $\widehat{u}$, and the contrast of $u$ is adjusted. Meanwhile the intrinsic textured information is contained in $v$, and the illumination effect in $v$ is compensated by QI.

Lastly, a feature fusion method is used to generate the image which has the illumination effect compensated:

$$
\widehat{I}=\alpha \widehat{u} \oplus \beta \widehat{v}
$$

where $\oplus$ is a fusion operator, $\alpha$ and $\beta$ are two fusion factors. There are many kinds of image fusion methods, and some are even based on fuzzy logic. In this paper we just choose a simple fusion method: $\oplus$ is chosen as a plus operator and $\alpha=\beta=1 / 2$. The fused result is just a weighted average of two parts. More complicated fusion method could be used in this model, and we will refer to it in further paper.

\subsection{Image Inpainting}

The image domain of $\widehat{I}$ in which all the illumination effect is compensated is denoted as $\boldsymbol{\Omega} \boldsymbol{\Omega}$ is composited as

$$
\boldsymbol{\Omega}=\mathbf{D} \cup \mathbf{D}^{\mathbf{c}},
$$

where $\mathbf{D}$ denotes the target region which needs to be restored, and the image information in $\mathbf{D}^{\mathbf{c}}$ is known. In this paper, the information in $\mathbf{D}$ is restored based on that in $\mathbf{D}^{\mathbf{c}} \mathbf{u s i n g}$ a joint-diffused PDEs model.

The geometric property and gray information are two factors that determine the property of an image, so a good inpainting model should restore them simultaneously, and this is just the joint diffusion implemented in this paper.

Two orthogonal geometric directions in image are shown in Fig. 2. $\Gamma$ is a gray curve in the image. Along the curve, pixels' gray values are consistent. $\xi$ denotes the isophotes

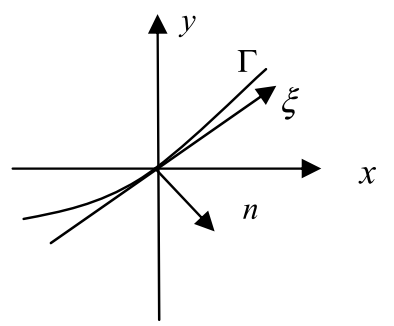

Fig. 2. Geometric directions in image. 
direction and $n$ denotes the gradient direction. Along the gradient direction, pixels' gray levels change greatly. The magnitude of a pixel's gradient vector equals to its changing rate of gray value. So by diffusing the gradient vectors, the geometric property of image is maintained. To preserve the discontinuities in image, a TV energy function about gradient vectors is used to diffuse image information:

$$
E(I)=\int_{D}|\nabla \overrightarrow{\mathbf{n}}| \mathrm{d} x,
$$

where $\overrightarrow{\mathbf{n}}$ denotes the gradient direction. The numerical form of Eq. (12) is

$$
\overrightarrow{\mathbf{n}}_{t}=\operatorname{div}(\nabla \overrightarrow{\mathbf{n}} /|\nabla \overrightarrow{\mathbf{n}}|)
$$

When the geometric property of gradient vectors is diffused, the geometric directions in the target region are updated. A Pixel's gray information is then diffused along the two updated geometric directions:

$$
\begin{aligned}
& I_{0}=\overparen{I}, \quad \omega_{0}=\Delta I_{0}, \quad \mathbf{u}_{0}=\nabla^{\perp} I_{0} \\
& \widehat{I}_{n+1}=\widehat{I}_{n}+\Delta t(\nabla \cdot(\nabla \overparen{I} /|\nabla \overparen{I}|)) \\
& \omega_{n+1}=\Delta \widehat{I}_{n+1}, \quad \mathbf{u}_{n+1}=\nabla^{\perp} \widehat{I}_{n+1}, \\
& \omega_{n+2}=\omega_{n+1}-\Delta t\left(\mathbf{u}_{n+1} \cdot \nabla w_{n+1}\right) \\
& \Delta \overparen{I}_{n+2}=\omega_{n+2} .
\end{aligned}
$$

Eqs. (14) is a bi-directional diffused model which is used to update gray information. It is a coupled model and there is no undetermined coefficient. The diffusion item which is along $\overrightarrow{\mathbf{n}}$ is $(\nabla \cdot(\nabla \widehat{I} /|\nabla \widehat{I}|))$ and the along isophotes diffusion item is $\left(\mathbf{u}_{n+1} \nabla w_{n+1}\right)$.

$(\nabla \cdot(\nabla \widehat{I} /|\nabla \widehat{I}|))$ equals to the curvature in image which is morphological invariant, so the stable status of TV model is adopted as the along gradient direction $\overrightarrow{\mathbf{n}}$ diffused item directly. The detailed analysis of TV model is given in (Chan and Shen, 2001a).

The along isophotes diffused item is derived according to the inviscid Helmholtz vorticity equation in fluid dynamics. In fluid dynamics, the well-known Navier-Stokes (N-S) equation for uncompressible fluid is

$$
\begin{aligned}
& \rho(\mathrm{d} \mathbf{u} / \mathrm{d} t)=\rho \mathbf{f}-\nabla p+v \Delta \mathbf{u} \\
& \mathbf{u}=\nabla^{\perp} \Psi
\end{aligned}
$$

where $\mathbf{u}$ is the fluid velocity, $\mathbf{f}$ is the external force in fluid, $\rho$ is the fluid density, $p$ is the pressure, $v$ is the viscosity coefficient, and $\Psi$ is the stream function in fluid. The velocity part can be written as

$$
\frac{\mathrm{d} \mathbf{u}}{\mathrm{d} t}=(\mathbf{u} \cdot \nabla) \mathbf{u}=\nabla\left(\frac{\mathbf{u} \cdot \mathbf{u}}{2}\right)-\mathbf{u} \times \omega
$$


where $\omega$ is vorticity in fluid. In vorticity fluid, the vorticity equals to the curl of velocity: $\omega=\nabla \times \mathbf{u}$.The curl result of both sides of N-S equation is

$$
\frac{\partial(\nabla \times \mathbf{u})}{\partial t}-\nabla \times(\mathbf{u} \times \omega)=\nabla \times f-\nabla \times\left(\frac{1}{\rho} \nabla p\right)+v \Delta(\nabla \times \mathbf{u}) .
$$

The vorticity continuity equation is

$$
\nabla \cdot \omega=\nabla \cdot(\nabla \times \mathbf{u})=0 .
$$

Expanding the curl part, then we get the Helmholtz vorticity equation:

$$
\mathrm{d} \omega / \mathrm{d} t=\partial \omega / \partial t+(\mathbf{u} \cdot \nabla) \omega=(\omega \cdot \nabla) \mathbf{u}+\nabla \times f-\nabla \times\left(\frac{1}{\rho} \nabla p\right)+v \Delta \omega .
$$

In 2-D image domain, $(\omega \cdot \nabla) \mathbf{u}, \nabla \times f, \nabla \times(\nabla p / \rho)$ are all zeros, so the Helmholtz equation can be simplified as

$$
\mathrm{d} \omega / \mathrm{d} t=v \Delta \omega
$$

Used in image inpainting, the Helmholtz equation should maintain the inviscid property (Bertalmio et al., 2001). The inviscid Helmholtz vorticity equation is

$$
\mathrm{d} \omega / \mathrm{d} t=0 .
$$

$\mathrm{d} \omega / \mathrm{d} t$ is the material derivative of $\omega$. It is composited as

$$
\mathrm{d} \omega / \mathrm{d} t=\partial \omega / \partial t+(\mathbf{u} \cdot \nabla) \omega
$$

Then the inviscid Helmholtz vorticity equation in 2-D image domain is

$$
\partial \omega / \partial t+(\mathbf{u} \cdot \nabla) \omega=0 \quad \Rightarrow \quad \partial \omega / \partial t=-(\mathbf{u} \cdot \nabla) \omega .
$$

The inviscid Helmholtz equation transports a smooth measure $\omega$ in fluid domain along the isophotes direction $\mathbf{u}$ and it is morphological invariant. So it is adopted as the along isophotes diffused item in the inpainting model.

The whole inpainting procedure is shown in Fig. 3. We restore the illumination compensated image $\widehat{I}$ using PDEs. Firstly the gray value is updated based on the TV model which diffuses along the gradient. Secondly the diffusion items for Eq. (23) are initialized: $\omega$ is computed as the smooth measure of image, and the movement direction of fluid is the isophote in image. Thirdly the vorticity and movement direction of fluid are updated, and $\omega$ is updated according to the Helmholtz equation. Then the value of $\omega$ is generated. To update all pixels' gray values according to $\omega$, it is necessary to solve the Poisson problem. It is proved that using a Poisson equation or its dynamic relaxation form 


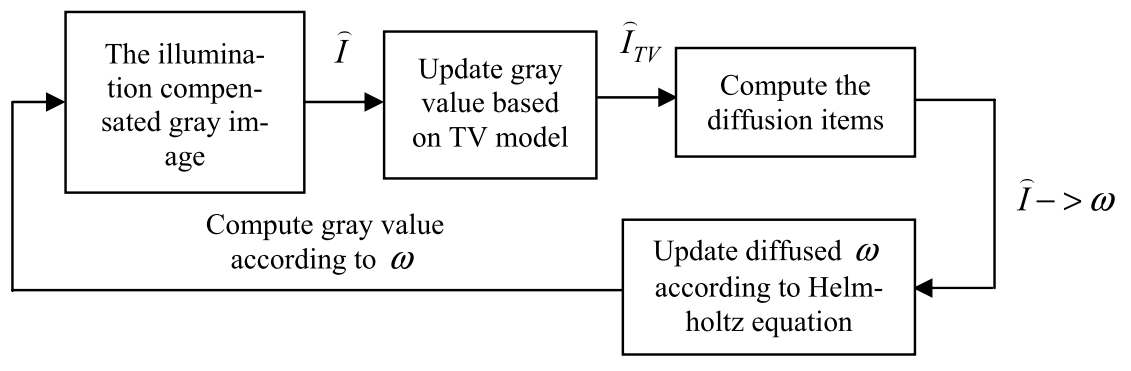

Fig. 3. The inpainting procedure.

does not affect the inpainting result (Bertalmio et al., 2001). To accelerate the diffusion procedure, the dynamic relaxation form is used in this paper:

$$
\widehat{I}_{t}=\alpha[\Delta \widehat{I}+\omega] .
$$

Finally gray values of all pixels are updated. During the inpainting procedure, the gray value and vorticity measure interact, $\widehat{I}_{0} \rightarrow \omega_{0} \rightarrow \widehat{I}_{1} \rightarrow \omega_{1} \rightarrow \cdots \rightarrow \widehat{I}_{n} \rightarrow \omega_{n}$. The procedure shown in Fig. 3 is executed circularly until the diffusion equations arrive at a stable status.

\section{Experiments}

In this section, different kinds of images are used to test the validity of the novel illumination compensation image inpainting model.

The benefit of the joint-diffused PDEs is proved firstly. Both the geometric property and gray information are diffused. The model is denoted as "JDM". Two PDE models which diffuse only along one geometric direction are used to compare with JDM. The model which diffuses along the gradient direction is denoted as "GDM" and the model which diffuses along isophotes is denoted as "IDM". SNR (Signal-to-Noise Ratio) of images processed by different inpainting models is shown in Table 1. The SNRs generated by the novel JDM are largest. Since it diffuses the geometric property and gray information simultaneously, and it diffuses the gray information along two orthogonal directions. The image processed by it is smooth and preserves the linear structure. The image processed by conventional models which diffuses along only one direction is not as good as that by the novel model.

Fig. 4 shows the inpainted images of different models. Fig. 4(a) is the results of the model which diffuses along isophotes. The model diffuses inward, so the whole region updates pixels' gray values simultaneously. The contour of target region is smoothed well, but the gray value of cobnut is error diffused. Fig. 4(b) gives the images inpainted by TV model. TV model diffuses along the gradient direction. So the diffusion procedure takes place from the top of rope to the bottom. There is no error diffusion and the whole region is inpainted well. But there is no diffusion across the contour of the target region, 
Table 1

SNRs of images processed by different inpainting models

\begin{tabular}{lccc}
\hline \multirow{2}{*}{ Original image } & \multicolumn{3}{c}{ Different processing models } \\
\cline { 2 - 4 } & JDM & GDM & IDM \\
\hline Image "Lincoln" & 36.9396 & 29.0259 & 33.8260 \\
& 34.6131 & 26.6370 & 33.1526 \\
& 29.5291 & 29.4020 & 28.9051 \\
\cline { 2 - 4 } Image "Squirrel" & 41.9456 & 40.8307 & 41.7700 \\
& 35.1318 & 23.1060 & 34.4607 \\
Image "Lena" & 29.3670 & 22.4191 & 29.2423 \\
\cline { 2 - 4 } & 40.4986 & 40.3759 & 39.3481 \\
& 45.4121 & 44.3803 & 39.8745 \\
& 32.8756 & 31.6903 & 32.8173 \\
\hline
\end{tabular}

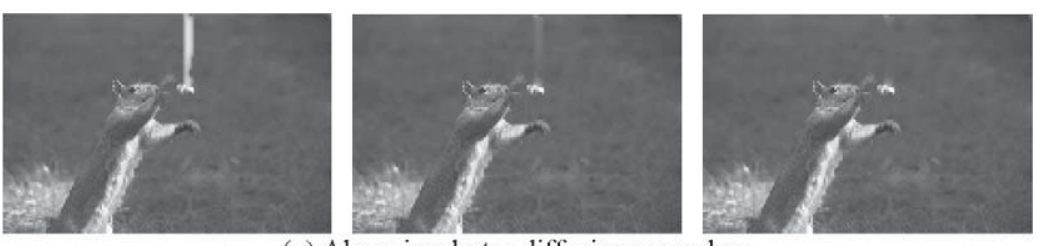

(a) Along isophotes diffusion procedure

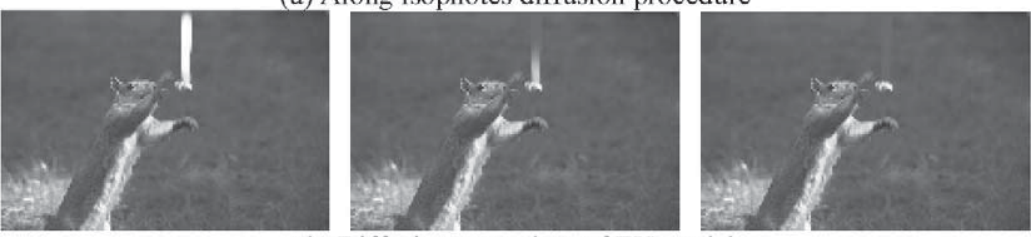

(b) Diffusion procedure of TV model

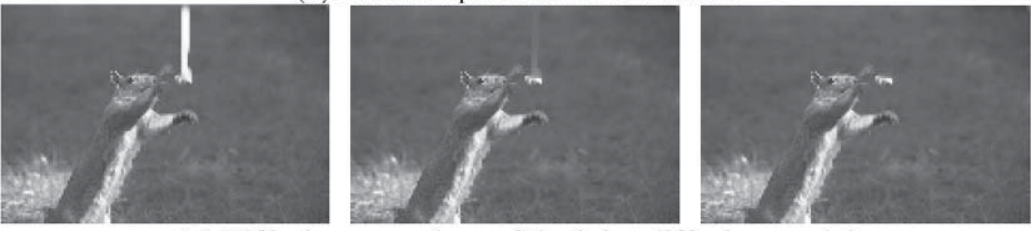

(c) Diffusion procedure of the joint-diffusion model

Fig. 4. Diffusion procedures of image "Squirrel" using different models.

so the contour is not smoothed. Fig. 4(c) shows images inpainted by the novel jointdiffused model. The diffusion procedure takes place at two orthogonal directions. The topper parts are inpainted earlier than the lower parts, while the information across the contour is diffused inward simultaneously. Therefore, the inpainted image is smooth. All the marked contour of the target region is not perceivable. There is no error diffusion in Fig. 4(c) and the visual perception of it is good.

The testing images in Fig. 5 are chosen from the CAS-PEAL face database (Gao et al., 2004). We added some scratches into the images under varying lighting. To prove the 


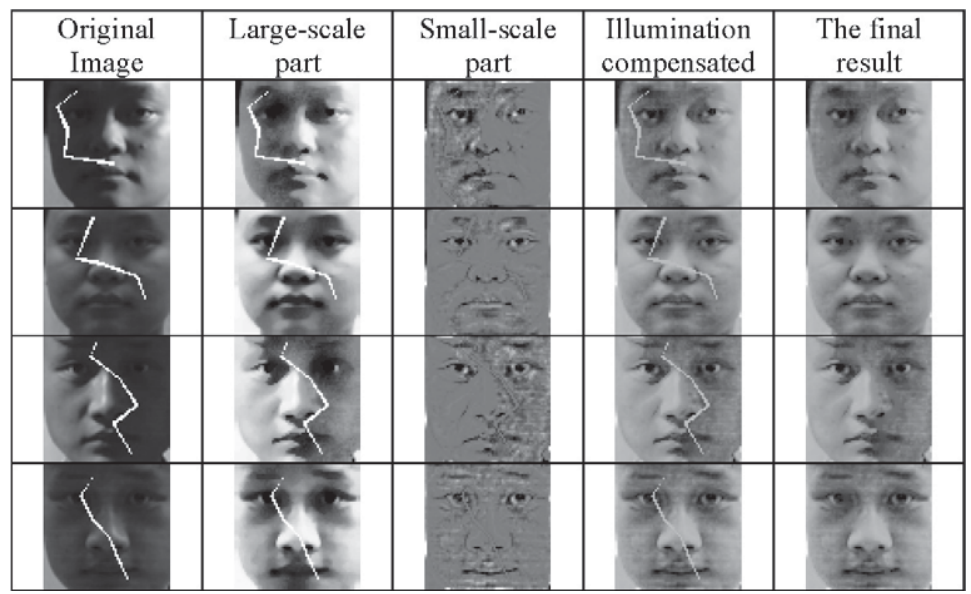

Fig. 5. Inpainting with illumination compensation in face images.

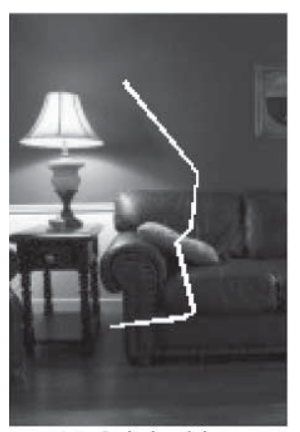

(a) Original image

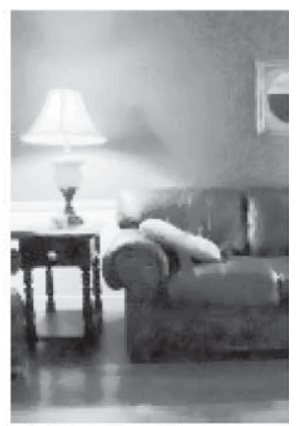

(b) Restored image

Fig. 6. Inpainting in the underexposure natural image.

effect of illumination compensation, the large-scale part and small-scale part of image are shown. The shadows and face contour are preserved in the large-scale part while the intrinsic textured information is contained in the small-scale part. All the image information is enhanced in the fused result of two parts. The scratch is well resorted in the final result. It is proved the scratched testing face images under varying lighting could be well restored by the novel model.

Lastly, two underexposure natural images are tested in Fig. 6 and Fig. 7. In the original images, the details of scenes are too dark to perceive, and there are scratches in them. In the restored images, illumination effects are compensated and the contrasts of the whole images are enhanced. Also, the default information in scratches is well restored. All the geometric structures and curvatures in the natural image are well preserved. The visual perceptions of the processed images are good. 


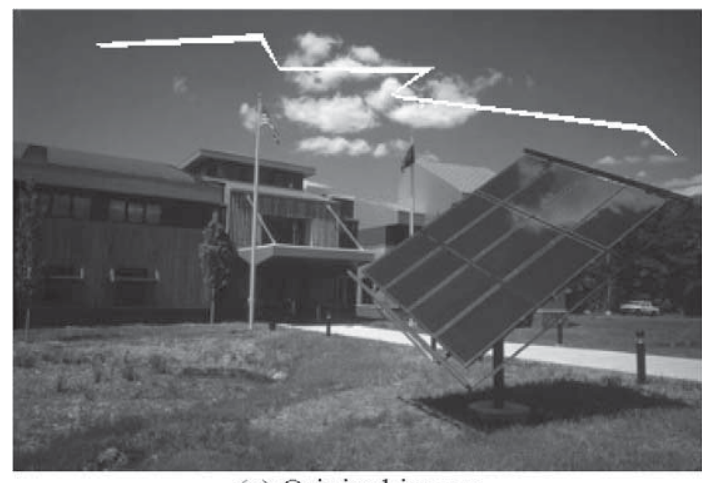

(a) Original image

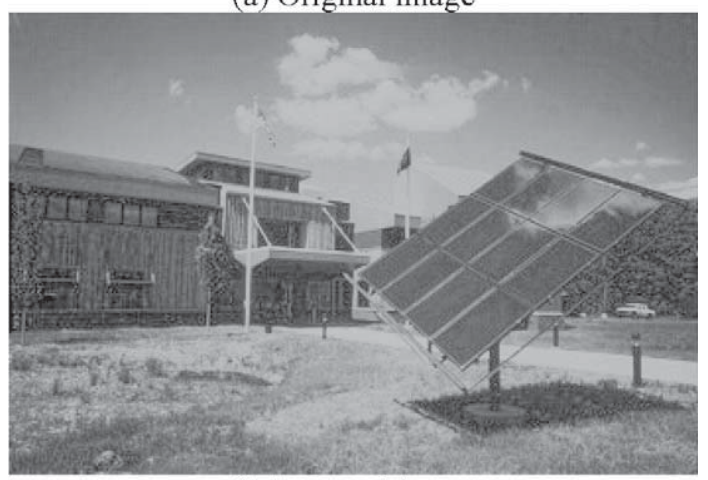

(b) Restored image

Fig. 7. Inpainting in the underexposure natural image.

\section{Conclusions}

In this paper a novel illumination compensation inpainting model which employs jointdiffused PDEs is proposed. It could restore the underexposure image directly. The model restores the lighting information and enhances image contrast in two scales, and then inpaint the illumination compensated image by diffusing the geometric property and gray information simultaneously. The joint-diffused PDEs restore the target region fast and precisely. They could well reconnect the geometric structure and restore the region smoothly. The model is tested in many images, and the result demonstrates the model could well inpaint underexposure image with shadows. The visual perception of the processed image is better than that of the conventional models.

\section{Acknowledgement}

The authors are grateful to the anonymous referees for their constructive and helpful comments. This paper is partly supported by the National Natural Science Foundation of China (No. 60472033, No. 60672062), the National Grand Fundamental Research 973 Program of China (No. 2004CB318005), the Technological Innovation Fund of Excellent Doctorial Candidate of Beijing Jiaotong University (No. 48026). 


\section{References}

Adini, Y., Y. Moses and S. Ullman (1997). Face recognition: the problem of compensating for changes in illumination direction. IEEE Transactions on Pattern Analysis and Machine Intelligence, 19(7), 721-732.

Ballester, C., M. Bertalmio, V. Caselles, G. Sapiro and J. Verdera (2001). Filling-in by joint interpolation of vector fields and gray levels. IEEE Transactions on Image Processing, 10(8), 1200-1211.

Bertalmio, M., A.L. Bertozi and G. Sapiro (2001). Navier-Stokes, fluid dynamics and image and video inpainting. In IEEE Conference on Computer Vision and Pattern Recognition.

Chan, T.F., and J. Shen (2001a). Mathematical models for local nontexture inpaintings. SIAM Journal on Applied Math, 62(3), 1019-1043.

Chan, T.F., and J. Shen (2001b). Morphologically invariant PDE inpaintings. UCLA CAM Report.

Chan, T.F., S.H. Kang and J. Shen (2002). Euler's elastica and curvature based inpainting. SIAM Journal of Applied Math, 63(2), 564-592.

Chen, T., W. Yin, X.S. Zhou, D. Comaniciu and T.S. Huang (2006). Total variation models for variable lighting face recognition. IEEE Transactions on Pattern Analysis and Machine Intelligence, 28, 1519-1524.

Gao, W., B. Cao, S. Shan, D. Zhou, X. Zhang and D. Zhao (2004). The CAS-PEAL large-scale Chinese face database and evaluation protocols. Technical Report No.JDL_TR_04_FR_001, Joint Research \& Development Laboratory, CAS.

Li, Z., and K. Jain (2005). Handbook of Face Recognition. Springer.

Malickas, A., and R. Vitkus (2001). Geometric invariants in image registration tasks. Informatica, 12(3), 385412.

Park, J.W., and K.H. Lee (2007). Inpainting highlights using color line project. IEICE Trans. Inf. \& Syst., E90D, 250-257.

Tai, X.C., S. Osher and R. Holm (2006). Image inpainting using a TV-Stokes equation. UCLA, CAM-Report06-01.

Yin, W., D. Goldfarb and S. Osher (2005). Image cartoon-texture decomposition and feature selection using the total variation regularized L1 functional. In Proceeding of Third International Workshop Variational, Geometric, and Level Set Methods in Computer Vision. pp. 73-84.

J. Wu was born in Hebei, China in 1982. She received the BS degree in computer science and technology from Beijing Jiaotong University, Beijing, China in 2004. She is currently pursuing the $\mathrm{PhD}$ degree at the Institute of Information Science, Beijing Jiaotong University. Her research interests include image processing, partial differential equation, wavelet analysis.

Q. Ruan was born in 1944. He received the BS and MS degrees from Northern Jiaotong University, China in 1969 and 1981 respectively. Form January 1987 to May 1990, he was a visiting scholar in the University of Pittsburgh, and the University of Cincinnati. Subsequently, he has been a visiting professor in USA for several times. He has published 2 books and more than 100 papers, and achieved a national patent. Now he is a professor, doctorate supervisor and the head of the Institute of Information Science, Beijing Jiaotong University. He is a senior member of IEEE. His main research interests include digital signal processing, computer vision, pattern recognition, and virtual reality etc.

G. An was born in Shandong, China in 1980. He received the BS degree in computer science and technology from Beijing Jiaotong University, Beijing, China in 2003. He is currently pursuing the $\mathrm{PhD}$ degree at the Institute of Information Science, Beijing Jiaotong University. His research interests include image processing, face recognition, independent component analysis, etc. 


\section{Tiesinę geometrinę struktūrą išsaugantis bendro skleidimo modelis} nepakankamo išlaikymo paveikslams rekonstruoti

Jiying WU, Qiuqi RUAN, Gaoyun AN

Bendro skleidimo dalinių išvestiniu lygtis naudojantis apšvietimo kompensavimo modelis pasiūlytas nepakankamo išlaikymo paveikslams rekonstruoti. Pirmiausia naujas modelis kompensuoja apšvietimo efektą nepakankamo išlaikymo paveiksluose. Tada informacija sulietame kompensuotame paveiksle yra rekonstruojama naudojant daliniu išvestiniu lygtis, skleidžiančias ị rekonstruojamą sritị kartu ir geometrines savybes, ir pilkumo informaciją. Eksperimentas demonstruoja, kad naujas modelis geli tinkamai rekonstruoti įbrèžimus kompensuodamas apšvietima, o bendro skleidimo dalinių išvestiniu lygtis yra tikslingiau naudoti negu bendrai priimtus rekonstravimo modelius. 